\title{
The success of extragalactic infrared interferometry: from what we have learned to what to expect
}

\author{
Konrad R. W. Tristram ${ }^{\mathrm{a}}$ and Sebastian F. Hönig ${ }^{\mathrm{b}}$ \\ ${ }^{a}$ European Southern Observatory, Alonso de Córdova 3107, Casilla 19001, Santiago, Chile

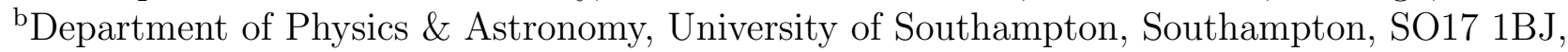
UK

\begin{abstract}
Infrared interferometry has lead to a breakthrough in the investigation of Active Galactic Nuclei (AGN) by allowing to resolve structures on sizes of less than a few parsecs in nearby galaxies. Measurements in the nearinfrared probe the innermost, hottest dust surrounding the central engine and the interferometrically determined sizes roughly follow those inferred from reverberation measurements. Interferometry in the mid-infrared has revealed parsec-sized, warm dust distributions with a clear two component structure: a disk-like component and polar emission - challenging the long-standing picture of the "dusty torus". New beam combiners are starting to resolve the kinematic structure of the broad line region and are expected to provide true images of the dust emission. Nevertheless, most AGN will remain only marginally resolved by current arrays and next generation facilities, such as the Planet Formation Imager (PFI), will be required to fully resolve out larger samples of AGN.
\end{abstract}

Keywords: Infrared Interferometry, galaxies: nuclei, galaxies: Seyfert, galaxies: active

\section{INTRODUCTION}

In astronomy, long baseline infrared interferometry is used to study compact thermal emission as well as compact line emission (e.g. using the $\operatorname{Br} \gamma$ line at 2.166 $\mu \mathrm{m}$ ). For the coherent combination of light from astronomical objects, sufficient signal to noise ratios are required in order to detect the fringes and measure their contrast. This is because the fringes are constantly shifting in phase and group delay, due to the turbulence in the Earth's atmosphere. As a consequence, when integrating longer than the coherence time ${ }^{1}$ the fringes get smeared out and hence become undetectable. In practice, this means only sufficiently bright astronomical sources can be observed, e.g. $\mathrm{K} \lesssim 11 \mathrm{mag}(\gtrsim 30 \mathrm{mJy})$ and $\mathrm{N} \lesssim 5 \mathrm{mag}(\gtrsim 200 \mathrm{mJy})$ for current technology. At the same time the sources have to have sizes $<100$ mas for interferometry to be a reasonable alternative to direct imaging using large telescopes in space or on the ground and equipped with adaptive optics.

To this date, for extragalactic sources only Active Galactic Nuclei (AGN) fulfil these requirements. In this paper the success of extragalactic infrared interferometry will be described. The paper is divided as follows: Sec. 2 gives an introduction into AGN; in Sec. 3, measurements leading to size estimates are discussed; Sec. 4 focuses on the results obtained from continuum studies going beyond size estimates; Sec. 5 focuses on a few detailed studies of individual sources; Sec. 6 presents prospects to use emission line studies; Sec. 7 reviews results using AGN variability as well as using interferometry to obtain distances; and finally Sec. 8 concludes with an outlook of what can be expected in the future for the field.

In total, AGN studies with 4 beam combining instruments at 2 different facilities, the Keck Interferometer and the Very Large Telescope Interferometer (VLTI) have been published, leading to 30 publications as of May 2018 (see table 1), with many more using the measurements or their results for further investigations or modelling.

Further author information: (Send correspondence to K.R.W.T.)

K.R.W.T.: E-mail: konrad.tristram@eso.org, Telephone: +56 224633156 
Table 1. List of long baseline optical interferometry publications of extragalactic sources

\begin{tabular}{|c|c|c|c|c|}
\hline First Author & Date & Objects & Interferometer & Band \\
\hline Swain et al. & $2003-10$ & NGC4151 & Keck & $\mathrm{K}$ band \\
\hline Wittkowski et al. & 2004-04 & NGC1068 & VINCI & $\mathrm{K}$ band \\
\hline Jaffe et al. & 2004-05 & NGC1068 & MIDI & $\mathrm{N}$ band \\
\hline Poncelet et al. & 2006-05 & NGC1068 & MIDI & $\mathrm{N}$ band \\
\hline Meisenheimer et al. & 2007-08 & Centaurus A & MIDI & $\mathrm{N}$ band \\
\hline Tristram et al. & $2007-11$ & Circinus & MIDI & $\mathrm{N}$ band \\
\hline Beckert et al. & 2008-08 & NGC3783 & MIDI & $\mathrm{N}$ band \\
\hline Kishimoto et al. & 2009-01 & NGC1068, Mrk1239, NGC3783, NGC4151 & Keck \& MIDI & $K \& N$ band \\
\hline Raban et al. & 2009-04 & NGC1068 & MIDI & $\mathrm{N}$ band \\
\hline Tristram et al. & 2009-07 & 8 targets ("Snapshot Survey") & MIDI & $\mathrm{N}$ band \\
\hline Burtscher et al. & 2009-11 & NGC4151 & MIDI & $\mathrm{N}$ band \\
\hline Kishimoto et al. & 2009-12 & 4 type 1 targets & Keck & $\mathrm{K}$ band \\
\hline Pott et al. & 2010-06 & NGC4151 & Keck & $\mathrm{K}$ band \\
\hline Burtscher et al. & $2010-10$ & Centaurus A & MIDI & $\mathrm{N}$ band \\
\hline Kishimoto et al. & 2011-03 & 8 type 1 targets & Keck & $\mathrm{K}$ band \\
\hline Tristram \& Schartmann & 2011-07 & 10 targets ("Snapshot Survey") & MIDI & $\mathrm{N}$ band \\
\hline Kishimoto et al. & 2011-12 & 6 type 1 targets & MIDI & $\mathrm{N}$ band \\
\hline Weigelt et al. & 2012-05 & NGC3783 & AMBER & $\mathrm{K}$ band \\
\hline Petrov et al. & 2012-07 & $3 \mathrm{C} 273$ & AMBER & $\mathrm{K}$ band \\
\hline Hönig et al. & 2012-08 & NGC424 & MIDI & $\mathrm{N}$ band \\
\hline Hönig et al. & 2013-07 & NGC3783 & MIDI & $\mathrm{N}$ band \\
\hline Burtscher et al. & 2013-10 & 23 targets ("AGN Large Programme") & MIDI & $\mathrm{N}$ band \\
\hline Kishimoto et al. & $2013-10$ & NGC4151 \& 5 further type 1 targets & Keck & $\mathrm{K}$ band \\
\hline Tristram et al. & 2014-03 & Circinus & MIDI & $\mathrm{N}$ band \\
\hline López-Gonzaga et al. & 2014-05 & NGC1068 & MIDI & $\mathrm{N}$ band \\
\hline López-Gonzaga et al. & 2016-06 & 23 targets ("AGN Large Programme") & MIDI & $\mathrm{N}$ band \\
\hline GRAVITY Collaboration & 2017-06 & PDS456 & GRAVITY & $\mathrm{K}$ band \\
\hline Fernández-Ontiveros & 2018-03 & IC3639 & MIDI & $\mathrm{N}$ band \\
\hline Leftley et al. & $2018-06$ & ESO323-G77 & MIDI & $\mathrm{N}$ band \\
\hline
\end{tabular}

\section{ACTIVE GALACTIC NUCLEI}

AGN are the most luminous persistent light sources in the universe, with high energy outputs sustained for millions of years. The large amounts of energy released in the nucleus have a significant influence on the surrounding galaxy and the intergalactic medium, e.g. preventing the gas from cooling and by quenching star formation in the host galaxy. The influence of the AGN on its environment is often referred to as "AGN feedback". AGN are therefore believed to play a fundamental role for galaxy evolution, that is for the build-up of galaxies. ${ }^{2}$

The driving engine responsible for the energy release in an AGN is accretion onto the supermassive black hole at the centre of a galaxy. Indeed most massive galaxies host a central black hole with a mass between $10^{6}$ 
and $10^{9} \mathrm{M}_{\odot}$. The result of the accretion process are jets and radiation over the entire electromagnetic spectrum from x-rays to radio wavelengths. The emission has a peak in the infrared, the "IR bump", which is thought to come from dust reprocessing the light from the central accretion disk and its corona which mainly radiate in $\mathrm{x}$-rays and the ultra violet ("big blue bump"). The main components of and AGN in the standard picture are hence: (1) a central accretion disk around the supermassive black hole on scales of $\lesssim 0.001$ pc; (2) high velocity, ionised gas emitting broad (up to several thousand $\mathrm{m} / \mathrm{s}$ ) emission lines in the so-called Broad Line Region (BLR) on scales of $\sim 0.01$ to $\sim 0.1 \mathrm{pc}$; (3) warm dust and molecular gas on scales of a few parsecs responsible for the infrared emission; (4) more distant, typically outflowing gas in ionisation cones or the so-called Narrow Line Region (NLR) reaching out to kiloparsec scales; as well as (5) relativistic jets of plasma probably launched close to the accretion disk and extending out to tens or hundreds of kiloparsecs.

AGN can be classified in two different types, those showing both broad and narrow emission lines ("type 1" / "Seyfert 1") and those only showing narrow emission lines ("type 2" / "Seyfert 2"). The discovery of "hidden" broad emission lines in polarised (that is scattered) light for NGC1068 ${ }^{3}$ gave birth to the unified scheme of AGN.",5 The idea is essentially that there is a type 1 nucleus hidden in each type 2 nucleus: the warm dust and gas have a toroidal distribution, the "dusty and molecular torus", leading to a viewing angle dependent obscuration of the central engine. When seen face on, our view towards the accretion disk and BLR are unobscured (type 1 case), when seen edge on the central regions are obscured.

The angular sizes of the BLR and the torus are very small and appear unresolved in single dish observations down to 100 mas even for the closest active nuclei. ${ }^{6}$ Information about the morphology and kinematics of this material can therefore only be inferred indirectly, e.g. by modelling of the Spectral Energy Distribution (SED) or by analysing the variability of the emitted radiation, especially reverberation mapping of the BLR and the hottest dust in the near-infrared. However any interpretations will remain highly ambiguous.

Only with interferometry is it possible to reach the angular scales required to resolve the inner regions of AGN on millarcsecond scales:

1. Using Very Large Baseline Interferometry (VLBI) measurements in the radio to observe water maser disks and outflows. ${ }^{7}$

2. Submm interferometry using ALMA tracing the warm molecular material. ${ }^{8-10}$

3. Infrared interferometry probing the dust emission as well as more recently the properties of line emitting gas such as in the BLR.

The main open questions are related to the physics of the accretion flow on parcsec scales, on the connection of inflow to outflow in the BLR and torus region, as well as the exact geometry and role of the nuclear obscurer.

\section{SIZE ESTIMATES}

The simplest quantity to measure using interferometry are sizes - in fact the first measurement interferometry was used for in astronomy was precisely to measure the sizes of stars, as first proposed by Fizeau in 1868 and carried out by Michelson \& Pease in $1921 .^{11}$ The same is true for optical interferometry of extragalactic sources. Because the thermal emission from the torus essentially remained unresolved in single-dish observation, only upper limits to its extent could be given and its true size remained unclear for a long time, with sizes up to hundreds of parsecs proposed.

The first size measurement of an AGN using infrared interferometry hence was a surprise. Using Keck Interferometer in the K-band, the near-infrared nuclear emission of the Seyfert 1 nucleus of NGC 4151 was found to be "unexpectedly compact", being only marginally resolved with a size of $\leq 0.1$ pc. ${ }^{12}$ As a consequence the emission was interpreted as mainly originating directly from the central accretion disk instead of hot dust close to sublimation. Shortly after, measurements of the Seyfert 2 galaxy NGC 1068 with the commissioning instrument VINCI at the VLTI, found larger sizes. Taking into account K-band speckle measurements, the data was interpreted in the form of a two component structure with a compact core of $<0.4 \mathrm{pc}$ and emission from larger scales on the order of $3 \mathrm{pc}$. 

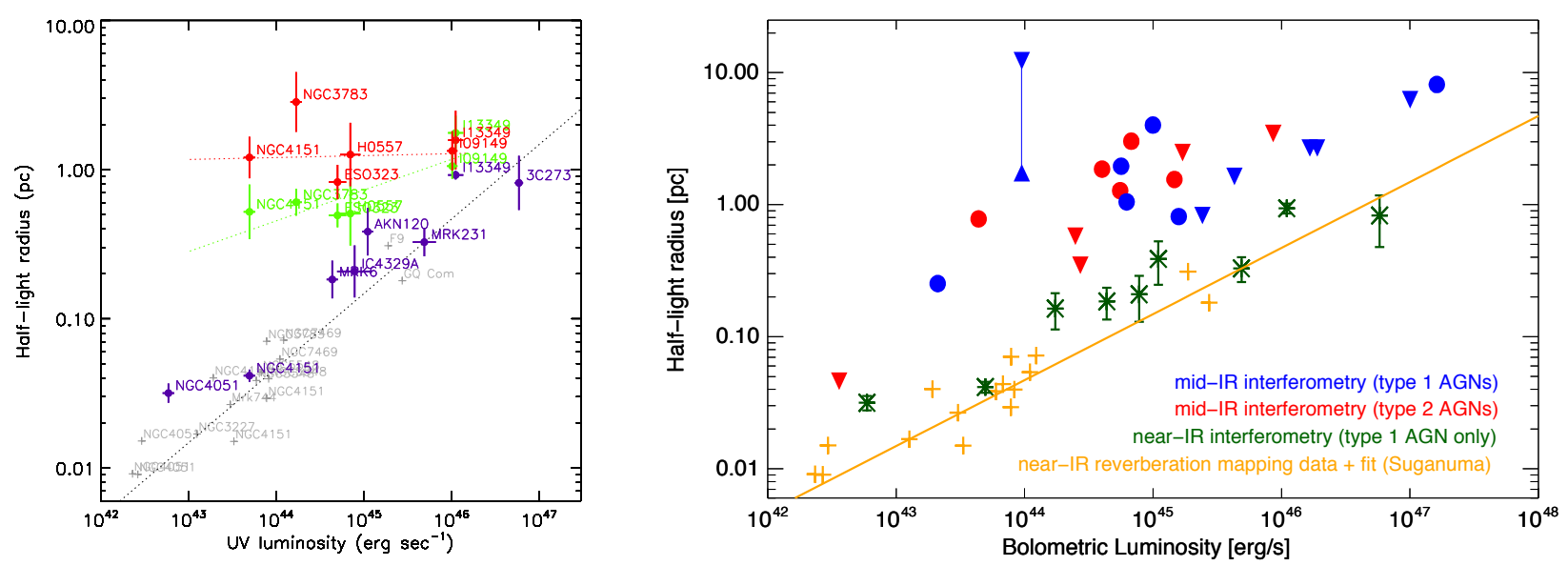

Figure 1. Size luminosity relations for the thermal emission around AGN from Refs. 13 (left, type 1 AGN only) and 14 (right): Reverberation sizes in grey (left) and orange (right); near infrared ring fit sizes in violet (left) and dark green (right). In the left panel the half light radii at 8.5 and $13 \mu \mathrm{m}$ are plotted, in the right panel the half light radii for type 1 (blue) and type 2 (red) AGN.

In the following years several size estimates for the dust emission could be obtained both in the K-band ${ }^{13,15,16}$ as well as in the N-band between $\lambda=8 \mu \mathrm{m}$ and $13 \mu \mathrm{m}^{14,17,18}$ Similar to what has been done for disks around Young Stellar Objects, ${ }^{19,20}$ the sizes can be plotted as a function of the intrinsic luminosity of the AGN, or rather a proxy for the latter such as the hard X-ray luminosity or the optical V-band luminosity in the case of unobscured type 1 objects. Examples from Refs. 13 and 14 are shown in Fig. 1. The K-band sizes show a relatively tight relation with $L^{1 / 2}$, where $L$ is the AGN luminosity, as is expected for centrally heated dust at the sublimation radius. The K-band sizes actually seem slightly larger than the sizes obtained from reverberation mapping, and the ratio of the two can be used as an approximate probe of the radial distribution of the material. ${ }^{16}$

In the mid-infrared, probing cooler dust further out, the picture is less clear: there are both claims that also the cooler dust roughly follows the $L^{1 / 2}$ relation $^{17,18}$ as well as claims that there is essentially no dependency on luminosity for the sizes at $\lambda=13 \mu \mathrm{m} .{ }^{13}$ The latter is interpreted as a sign of steeper dust distributions for more luminous sources. The most complete size-luminosity relation for the mid-infrared to date does show a general trend following $L^{1 / 2}$, with the mid infrared sizes 4 to 30 times larger than the relation determined by reverberation mapping. ${ }^{14}$ The large scatter implies that AGN tori are dominated by intrinsic differences in their dust structures. Most notably these differences are much large than the differences expected between type 1 and type 2 sources.

One issue when determining sizes from visibilities is that typically a specific model for the brightness distribution must be assumed, especially if there are only very few measurements at different spatial frequencies and visibilities are close to 0 or 1 , the latter being the case for most K-band measurements. For the K-band, mainly ring fits were performed, while in the mid-infrared often Gaussian or $r^{-\alpha}$ brightness distributions are assumed. The choice of the model can lead to significantly different size estimates. Only when the visibilities cover the range of $V$ 0.5, the model-independent half-light radius can be determined, as is the case for the radii shown in Fig. 1 .

\section{CONTINUUM MORPHOLOGIES}

From the previous section it becomes clear that a better understanding of the radial distribution of the emission in AGN would be highly desirable. After first claims of a possible common brightness distribution following a $r^{-2}$ powerlaw, ${ }^{21}$ it now rather seems that AGN tori are dominated by intrinsic differences in their dust structures. ${ }^{14}$ Moreover, many sources seem to possess a two component structure in the mid-infrared. For 18 out of 23 sources studied in Ref. 14, two nuclear components can be distinguished in radial fits: a compact component 
often appearing unresolved even on the longest interferometric baselines as well a more extended well resolved component. Surprising is the relatively high level of unresolved flux and its large scatter. The median "point source fraction" is $70 \%$ for type 1 and $47 \%$ for type 2 AGNs meaning that a large part of the flux is concentrated on scales $<5$ mas $(0.1-10 \mathrm{pc})$. For sources observed with similar spatial resolution, the unresolved flux varies from $20 \%-100 \%$, confirming once more that there are large intrinsic differences in the dust structures.

Going one step further in complexity we can ask if the radial distribution is the same in all position angles, or more simply speaking if the emission is elongated in a certain direction. Indeed from the classical picture of the dusty torus or thick disk in the unified scheme this is expected: for type 2 systems with a highly inclined torus, the infrared emission is anticipated to be elongated in the equatorial direction, while for type 1 systems with an almost face-on torus no significant elongation is anticipated. But here comes the probably biggest surprise of infrared interferometry for AGN research: this seems not to be the case.

To securely make any statements on the elongation of the emission, the source must be resolved to a significant fraction and a sufficient uv coverage has to be reached, covering a wide range of position angles. Finally, the measurement uncertainties must be small enough to be able to unambiguously constrain an elongation. It therefore took a couple of years after the first interferometric measurements to overcome these limitations and to date this has only been possible for measurements taken in the mid-infrared with the mid-infrared beam combiner MIDI at the VLTI.

The first indications for an extension in polar direction came from the modelling of the interferometry data on Seyfert 2 nucleus of NGC 1068, ${ }^{22}$ where the extended emission component was found to be extended in the direction of the nuclear jet. This was followed by the detection of polar dust in another type 2 source NGC $424^{23}$ and then also in the type 1 nucleus of NGC $3783 .{ }^{24}$ A more systematic search for elongated emission using all MIDI data published by 2016, resulted in clearly elongated structures in five out of seven sources where the uv plane was sufficient to constrain an elongation. ${ }^{23}$ From these five sources, three are type 2 , one type 1i, and one type 1. The observed axis ratios are typically around 2 and the elongations were found to be more or less (but not exactly) in the polar direction as inferred from ionisation cones or polarisation. The remaining two sources, both Seyfert 2 nuclei, are consistent with the emission being radially symmetric. Recently a further type 1 source, ESO 323-G77, has been confirmed to show strong polar elongation. ${ }^{25}$ That is the mid-infrared emission polar extended in 3 of 4 sources. In fact deep single-dish observations reveal that the mid-infrared emission is extended predominantly in polar direction also on $100 \mid p c$ scales. ${ }^{6,26}$

The physical origin of the polar emission is still unclear, but first models have been proposed, suggesting that the emission comes from dusty polar wind or a hollow outflow cone, possibly driven by radiation pressure. ${ }^{27,28}$ An increased modelling effort as well as further interferometric measurements will be necessary to fully pin down the origin of the thermal emission and its relation to the physics of the torus.

\section{SUBSTRUCTURES IN INDIVIDUAL SOURCES}

For the two brightest and best resolved AGN in the mid-infrared, the Seyfert 2 galaxies NGC1068 and the Circinus galaxy, interferometric measurements with MIDI allowed to obtain a more detailed understanding of the brightness distribution. For both sources, a large number of UV points, 152 for the Circinus galaxy and 40 for NGC1068, were obtained using both the Unit Telescopes as well as the Auxiliary Telescopes of the VLTI. MIDI being a two beam combiner and the absolute phase information being corrupted by the atmosphere (see Sect. 1) the data had to be modelled. For this purpose, the correlated fluxes and chromatic phases measured by MIDI were fitted by a model consisting of black-body emitters with elongated Gaussian brightness distributions and with dust extinction. The resulting best fit model images are shown in Fig. 2.

Both sources reveal a clear two component structure composed of an inner disk-like component as well as a dust distribution extended perpendicular to it on scales of 2 to 3 pc (see Fig. 2). Also in several other aspects the results are very similar: The dust emission is not directly concentrated along the system axis as defined by the ionisation cone, but rather trace one of the cone edges. A strong gradient in the $13.0 \mu \mathrm{m}$ silicate absorption feature is observed in direction of the system axis and in agreement to the larger scale structures such as the one-sided ionisation cones (the other cones being obscured). The dense disk-like components only contribute to about one fourth of the total mid-infrared emission coming from the nuclei; the majority of the emission comes 

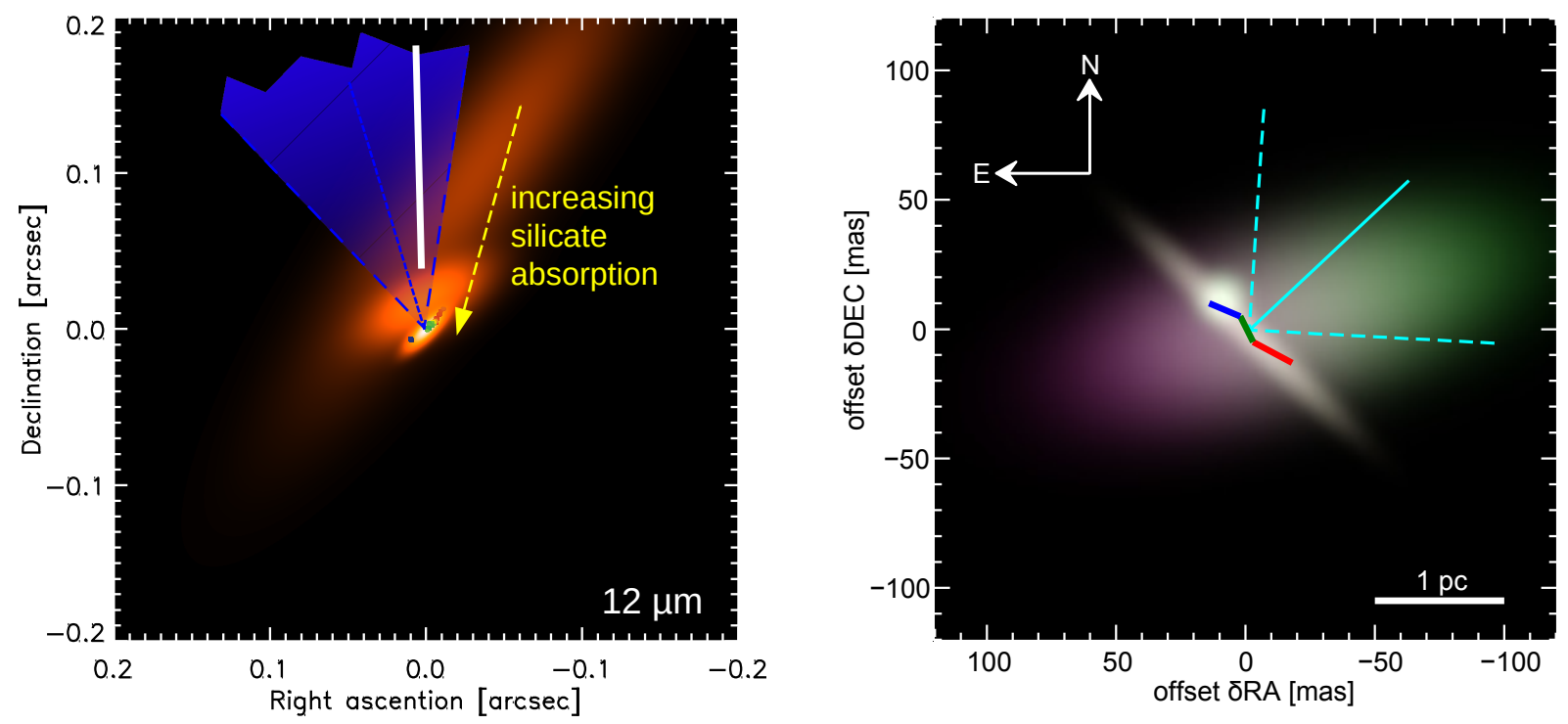

Figure 2. Model images for NGC1068 (left) and the Circinus galaxy (right) from Refs. 29 and 30 . The image for NGC 1068 shows the model brightness distribution of the mid-infrared emission at $12 \mu \mathrm{m}$. To set the dust emission in context, the coloured points in the very nucleus delineate the nuclear water maser disk, the thick white line indicates the direction of the nuclear jet and the blue cone represents the direction and opening angle of the ionisation cone on arcsec scales. The depth of the silicate absorption increases in the three components from North to South. The image for the Circinus galaxy is a false-colour image of the model brightness distribution. The colours red, green and blue correspond to the model at $13.0 \mu \mathrm{m}, 10.5 \mu \mathrm{m}$ and $8.0 \mu \mathrm{m}$, respectively. The colour gradient of the extended component is due to the increase in the silicate depth towards the south-east. Also plotted is the trace of the water maser disk: the blue and red parts trace the approaching and receding sides of the maser disk respectively.

from the extended components. Furthermore the disk-like components in both galaxies match water maser disks in orientation and size. On the other hand there are also a few subtle differences: While in NGC 1068 the temperature $T \sim 700 \mathrm{~K}$ of the disk-like component is significantly higher than that of the extended emission with $T \sim 300 \mathrm{~K}$, as to be expected for centrally heated dust, this is not the case for the Circinus galaxy. In that galaxy the disk-like component is at similarly low temperatures of $T \sim 300 \mathrm{~K}$ as the ore extended dust, somewhat difficult to explain for centrally heated dust. Furthermore the silicate feature of the disk component is significantly deeper towards the disk-like component for NGC 1068, while for the Circinus galaxy the opposite is the case.

A third source, the Seyfert 1 galaxy NGC 3783, is much less resolved than NGC1068 and the Circinus galaxy but nevertheless appears to show a similar structure. The emission comes from two distinct components, one hot component possibly elongated in the equatorial plane as traced by near-infrared interferometry and one warm extended in the polar direction as revealed by mid-infrared interferometry. ${ }^{24}$ These results are in good agreement to those found for the less resolved sources, especially concerning the two components structures and the polar extended emission.

\section{EMISSION LINES}

Apart from the continuum emission, also emission lines can be resolved interferometrically and spectrally. These are of special interest as they carry kinematic information about the material. Unfortunately, detailed spectrointerferometric studies such as those for e.g. Be stars $^{31}$ have not been possible for AGN due to the lack of sensitivity.

In the context of AGN, the most promising and also scientifically interesting target is to study the BLR using the $\operatorname{Br} \gamma$ or (for sufficiently distant and hence redshifted targets) the $\mathrm{Pa} \alpha$ line in the K-band. Because 
the BLR is much smaller - on the $\mu$ as scale - than what can be resolved by current interferometers, differential measurements of the visibilities and especially the phases as a function of the wavelength have to be carried out.

A first such measurement was carried out for the $\mathrm{Pa} \alpha$ line of the quasar 3C273 using the instrument AMBER at the VLTI. ${ }^{32}$ A novel observation method not requiring the real-time correction of the atmospheric fringe movements was used and a special data processing based on the accumulation of 2D Fourier amplitudes had to be employed. The measurements suggest a decrease in the differential visibility between the emission line and the continuum as well as a differential phase smaller than $3^{\circ}$. This would imply an angular radius of the BLR of more than 0.4 mas, somewhat in contradiction to 3 times smaller size estimates obtained from reverberation mapping. ${ }^{33}$

A second, measurement, also for the $\mathrm{Pa} \alpha$ line, was achieved with GRAVITY for PDS456, a quasar at $z=0.184 .^{34}$ The continuum appears partially resolved, with a best Gaussian fit FWHM size of 0.3 mas, which is comparable to the angular size expected for the dust sublimation radius. However, no differential phase or amplitude signature could be detected at the $\operatorname{Pa} \alpha$ line, despite a precision of $\lesssim 0.1^{\circ}$ for the differential phase on each of the two longest baselines. This constrains the line centroid displacement to $\lesssim 10 \mu$ as. Using the measured line strength and assuming ordered rotation or outflow, this places an upper limit on the offset of the line emission from the continuum of $\lesssim 150 \mu$ as. Further measurements have been carried out with GRAVITY in the meantime for this source, as well as for 3C273 and it seems that a signal has been detected for $3 \mathrm{C} 273$.

\section{VARIABILITY}

Due to the stochastic nature of accretion onto the supermassive black hole, the activity of AGN is variably on various time-scales. An increase in the luminosity of the accretion disk will lead to an increase of the emission from the BLR and of the thermal emission from the dust with different delays and time scales. This is used by reverberation mapping to infer the properties of these components. ${ }^{35,36}$ Moreover, for an increased luminosity $L$ of the accretion disk the corresponding BLR radius and the dust sublimation radius should increase with $r \propto L^{1 / 2}$. Indeed such size changes have been measured using reverberation mapping although these do not directly follow $L^{1 / 2}$ relation. ${ }^{37,38}$

While reverberation mapping can only deliver averaged sizes for for certain periods of time (100 days for the dust), interferometric measurements allow to determine the size of the dust and possibly the BLR instantaneously. So far, only one object has been studied in this respect using optical interferometry: NGC 4151. K-band measurements obtained with the Keck Interferometer as well as AMBER at the VLTI between 2003 and 2012 revealed that the size of the near-infrared emission structure does not strictly depend on the respective momentary nuclear luminosity. Instead it seems to correlate with a long-term average of the flux of the central engine over the previous several $(\sim 6)$ years. ${ }^{39,40}$ This implies that the destruction and reformation timescale of the innermost dust distribution is on such time scales, in agreement with the results from reverberation measurements. ${ }^{38}$

The possibility to determine angular sizes using optical interferometry and physical sizes using reverberation mapping allows to measure directly the angular-diameter distance to the AGN using the relation $D(\mathrm{Mpc})=$ $0.173 \tau$ (days) $/(\rho$ (mas) $(1+z))$, where $D$ is the distance, $\tau$ is the time lag obtained by reverberation measurements and $\rho$ is the angular diameter from interferometry. Originally proposed using the broad emission lines ("quasar parallax"), ${ }^{41}$ it was adapted and proven feasible using the hot dust emission ("dust parallax"): ${ }^{42}$ using K-band sizes and lags for NGC 4151, a distance of $D \sim 19 \mathrm{Mpc}$ could be determined, at the same time leading to a more accurate black hole mass estimate. If applied to other galaxies, this geometric-distance method could lead to an improvement in estimates of black-hole masses from reverberation mapping as well as direct distance measurements out to higher redshifts, allowing to determine cosmological properties.

We close with the note, that variability has also been observed in the mid-infrared, with flux changes up to a factor of 2 , which could also imply changes in the size of the emitting region by a factor of 2 . However the interferometric measurements do not seem affected by the flux changes, leaving it unclear from where the flux changes actually originate. Infrared monitoring campaigns and more rapidly carried out interferometric measurements will be required to shed more light on these variations. 


\section{CONCLUSION AND OUTLOOK}

Originally unclear if feasible, optical interferometry of AGN has become mature and provided a breakthrough for the investigation of the heated dust structures in AGN through continuum studies in the near- and mid-infrared. Using the interferometric measurements it could be shown that the innermost, hot dust mainly emitting in the K band scales with $L^{1 / 2}$ at radii close to the sublimation radius and only slightly larger than the radii determined from reverberation mapping. Dust destruction and formation timescale in this region are on the order of a few years. The cooler dust dominating the emission in the mid-infrared on the other hand, is located at ten times larger radii, but still on scales of less than $10 \mathrm{pc}$ with a large scatter interpreted as evidence for substantial intrinsic differences in their dust distributions. In contrast to model predictions for the emission from the dusty torus, the emission turned out to be extended in the polar direction. This has triggered new models being proposed and being tested using the interferometric data. However the fundamental question still remains open: Where does the main obscuration of the central source and the BLR emission take place and how is is the absorbed energy re-emitted.

Spectro-interferometry of AGN is still in its infancy but expectations are that GRAVITY with its exquisite sensitivity and stability will be able to resolve the kinematic structure of the broad line region. The combination of interferometric with reverberation measurements allows to determine direct quasar-parallax or dust-parallax distances to sources at significant redshift, obliterating the uncertainties of the cosmic distance ladder.

One limitation of the observations so far have is that it was not possible to reconstruct images for them, less for the near-infrared where the sources are barely resolved, more for the mid-infrared where several sources are well resolved. This will now change with the advent of MATISSE the second generation mid-infrared interferometer of the VLTI. The extragalactic interferometry community is eagerly looking forward to the first true images reconstructed from observations with this instrument expected to be appear at the end of 2018 and beginning of 2019 .

Unfortunately, there have been so far only 2 interferometric facilities that were sensitive enough to observe AGN: the Keck Interferometer as well as the VLTI, both with baseline lengths limited to $<130 \mid m$. CHARA is still fighting and once equipped with new AO fully operational hopefully able to lock on the faint extragalactic fringes. With baselines up to $330 \mathrm{~m}$ this would for the first time allow to also properly resolve the emission from the hot dust at the inner rim for the first time and allow to go beyond measuring ring fit radii. The main issues for extragalactic interferometry, sensitivity and unresolvedness. will only be overcome with a new array, ideally with kilometer-long baselines. A promising proposal is the Planet Formation Imager (PFI) and extragalactic science is among the more prominent secondary science cases. ${ }^{43}$

\section{REFERENCES}

[1] Kellerer, A. and Tokovinin, A., "Atmospheric coherence times in interferometry: definition and measurement," A\&A 461, 775-781 (Jan. 2007).

[2] Cattaneo, A., Faber, S. M., Binney, J., Dekel, A., Kormendy, J., Mushotzky, R., Babul, A., Best, P. N., Brüggen, M., Fabian, A. C., Frenk, C. S., Khalatyan, A., Netzer, H., Mahdavi, A., Silk, J., Steinmetz, M., and Wisotzki, L., "The role of black holes in galaxy formation and evolution," Nature 460, 213-219 (July 2009).

[3] Antonucci, R. R. J. and Miller, J. S., "Spectropolarimetry and the nature of NGC 1068," ApJ 297, 621-632 (Oct. 1985).

[4] Antonucci, R., "Unified models for active galactic nuclei and quasars," ARA $\& A$ 31, 473-521 (1993). Review article about the unified models of active galactic nuclei, which explain "much of the variety in AGN types is just the result of varying orientation relative to the line of sight.".

[5] Urry, C. M. and Padovani, P., "Unified Schemes for Radio-Loud Active Galactic Nuclei," PASP 107, 803 (Sept. 1995).

[6] Asmus, D., Hönig, S. F., Gandhi, P., Smette, A., and Duschl, W. J., "The subarcsecond mid-infrared view of local active galactic nuclei - I. The N- and Q-band imaging atlas," MNRAS 439, 1648-1679 (Apr. 2014).

[7] Lo, K. Y., "Mega-Masers and Galaxies," ARA\& A 43, 625-676 (Sept. 2005). 
[8] García-Burillo, S., Combes, F., Ramos Almeida, C., Usero, A., Krips, M., Alonso-Herrero, A., Aalto, S., Casasola, V., Hunt, L. K., Martín, S., Viti, S., Colina, L., Costagliola, F., Eckart, A., Fuente, A., Henkel, C., Márquez, I., Neri, R., Schinnerer, E., Tacconi, L. J., and van der Werf, P. P., "ALMA Resolves the Torus of NGC 1068: Continuum and Molecular Line Emission," ApJ 823, L12 (May 2016).

[9] Gallimore, J. F., Elitzur, M., Maiolino, R., Marconi, A., O’Dea, C. P., Lutz, D., Baum, S. A., Nikutta, R., Impellizzeri, C. M. V., Davies, R., Kimball, A. E., and Sani, E., "High-Velocity Bipolar Molecular Emission from an AGN Torus," ArXiv e-prints (Aug. 2016).

[10] Alonso-Herrero, A., Pereira-Santaella, M., García-Burillo, S., Davies, R. I., Combes, F., Asmus, D., Bunker, A., Díaz-Santos, T., Gandhi, P., González-Martín, O., Hernán-Caballero, A., Hicks, E., Hönig, S., Labiano, A., Levenson, N. A., Packham, C., Ramos Almeida, C., Ricci, C., Rigopoulou, D., Rosario, D., Sani, E., and Ward, M. J., "Resolving the nuclear obscuring disk in the Compton-thick Seyfert galaxy NGC5643 with ALMA," ArXiv e-prints (Apr. 2018).

[11] Michelson, A. A. and Pease, F. G., "Measurement of the Diameter of $\alpha$ Orionis with the Interferometer.," ApJ 53 (May 1921). Paper describing the first successful interferometric observations for astronomy.

[12] Swain, M., Vasisht, G., Akeson, R., Monnier, J., Millan-Gabet, R., Serabyn, E., Creech-Eakman, M., van Belle, G., Beletic, J., Beichman, C., Boden, A., Booth, A., Colavita, M., Gathright, J., Hrynevych, M., Koresko, C., Le Mignant, D., Ligon, R., Mennesson, B., Neyman, C., Sargent, A., Shao, M., Thompson, R., Unwin, S., and Wizinowich, P., "Interferometer Observations of Subparsec-Scale Infrared Emission in the Nucleus of NGC 4151," ApJ 596, L163-L166 (Oct. 2003).

[13] Kishimoto, M., Hönig, S. F., Antonucci, R., Millour, F., Tristram, K. R. W., and Weigelt, G., "Mapping the radial structure of AGN tori," $A \mathscr{E} A$ 536, A78 (Dec. 2011).

[14] Burtscher, L., Meisenheimer, K., Tristram, K. R. W., Jaffe, W., Hönig, S. F., Davies, R. I., Kishimoto, M., Pott, J.-U., Röttgering, H., Schartmann, M., Weigelt, G., and Wolf, S., "A diversity of dusty AGN tori. Data release for the VLTI/MIDI AGN Large Program and first results for 23 galaxies," A $6 A$ 558, A149 (Oct. 2013).

[15] Kishimoto, M., Hönig, S. F., Antonucci, R., Kotani, T., Barvainis, R., Tristram, K. R. W., and Weigelt, G., "Exploring the inner region of type 1 AGNs with the Keck interferometer," $A$ EA 507, L57-L60 (Dec. 2009).

[16] Kishimoto, M., Hönig, S. F., Antonucci, R., Barvainis, R., Kotani, T., Tristram, K. R. W., Weigelt, G., and Levin, K., "The innermost dusty structure in active galactic nuclei as probed by the Keck interferometer," $A \xi A$ 527, A121 (Mar. 2011).

[17] Tristram, K. R. W., Raban, D., Meisenheimer, K., Jaffe, W., Röttgering, H., Burtscher, L., Cotton, W. D., Graser, U., Henning, T., Leinert, C., Lopez, B., Morel, S., Perrin, G., and Wittkowski, M., "Parsec-scale dust distributions in Seyfert galaxies. Results of the MIDI AGN snapshot survey," A\&A 502, 67-84 (July 2009).

[18] Tristram, K. R. W. and Schartmann, M., "On the size-luminosity relation of AGN dust tori in the midinfrared," A\&A 531, A99 (July 2011).

[19] Monnier, J. D. and Millan-Gabet, R., "On the Interferometric Sizes of Young Stellar Objects," ApJ 579, 694-698 (Nov. 2002).

[20] Kraus, S., "The interferometric view of Herbig Ae/Be stars," Ap\&SS 357, 97 (June 2015).

[21] Kishimoto, M., Hönig, S. F., Tristram, K. R. W., and Weigelt, G., "Possible evidence for a common radial structure in nearby AGN tori," A\&A 493, L57-L60 (Jan. 2009).

[22] Raban, D., Jaffe, W., Röttgering, H., Meisenheimer, K., and Tristram, K. R. W., "Resolving the obscuring torus in NGC 1068 with the power of infrared interferometry: revealing the inner funnel of dust," MNRAS 394, 1325-1337 (Apr. 2009).

[23] Hönig, S. F., Kishimoto, M., Antonucci, R., Marconi, A., Prieto, M. A., Tristram, K., and Weigelt, G., "Parsec-scale Dust Emission from the Polar Region in the Type 2 Nucleus of NGC 424," ApJ 755, 149 (Aug. 2012).

[24] Hönig, S. F., Kishimoto, M., Tristram, K. R. W., Prieto, M. A., Gandhi, P., Asmus, D., Antonucci, R., Burtscher, L., Duschl, W. J., and Weigelt, G., "Dust in the Polar Region as a Major Contributor to the Infrared Emission of Active Galactic Nuclei," ApJ 771, 87 (July 2013). 
[25] Leftley, J., "ESO323-G77," A\&\&A (June 2018).

[26] Asmus, D., Hönig, S. F., and Gandhi, P., "The Subarcsecond Mid-infrared View of Local Active Galactic Nuclei. III. Polar Dust Emission," ApJ 822, 109 (May 2016).

[27] Hönig, S. F. and Kishimoto, M., "Dusty Winds in Active Galactic Nuclei: Reconciling Observations with Models," ApJ 838, L20 (Apr. 2017).

[28] Stalevski, M., Asmus, D., and Tristram, K. R. W., "Dissecting the active galactic nucleus in Circinus - I. Peculiar mid-IR morphology explained by a dusty hollow cone," MNRAS 472, 3854-3870 (Dec. 2017).

[29] López-Gonzaga, N., Jaffe, W., Burtscher, L., Tristram, K. R. W., and Meisenheimer, K., "Revealing the large nuclear dust structures in NGC 1068 with MIDI/VLTI," A\&̈A 565, A71 (May 2014).

[30] Tristram, K. R. W., Burtscher, L., Jaffe, W., Meisenheimer, K., Hönig, S. F., Kishimoto, M., Schartmann, M., and Weigelt, G., "The dusty torus in the Circinus galaxy: a dense disk and the torus funnel," $A \& \exists A \mathbf{5 6 3}$, A82 (Mar. 2014).

[31] Meilland, A., Millour, F., Kanaan, S., Stee, P., Petrov, R., Hofmann, K.-H., Natta, A., and Perraut, K., "First spectro-interferometric survey of Be stars. I. Observations and constraints on the disk geometry and kinematics," AËA 538, A110 (Feb. 2012).

[32] Petrov, R. G., Millour, F., Lagarde, S., Vannier, M., Rakshit, S., Marconi, A., and weigelt, G., "VLTI/AMBER differential interferometry of the broad-line region of the quasar 3C273," in [Optical and Infrared Interferometry III], Proc. SPIE 8445, 84450W (July 2012).

[33] Kaspi, S., Smith, P. S., Netzer, H., Maoz, D., Jannuzi, B. T., and Giveon, U., "Reverberation Measurements for 17 Quasars and the Size-Mass-Luminosity Relations in Active Galactic Nuclei," ApJ 533, 631-649 (Apr. 2000).

[34] Gravity Collaboration, Abuter, R., Accardo, M., Amorim, A., Anugu, N., Ávila, G., Azouaoui, N., Benisty, M., Berger, J. P., Blind, N., Bonnet, H., Bourget, P., Brandner, W., Brast, R., Buron, A., Burtscher, L., Cassaing, F., Chapron, F., Choquet, É., Clénet, Y., Collin, C., Coudé Du Foresto, V., de Wit, W., de Zeeuw, P. T., Deen, C., Delplancke-Ströbele, F., Dembet, R., Derie, F., Dexter, J., Duvert, G., Ebert, M., Eckart, A., Eisenhauer, F., Esselborn, M., Fédou, P., Finger, G., Garcia, P., Garcia Dabo, C. E., Garcia Lopez, R., Gendron, E., Genzel, R., Gillessen, S., Gonte, F., Gordo, P., Grould, M., Grözinger, U., Guieu, S., Haguenauer, P., Hans, O., Haubois, X., Haug, M., Haussmann, F., Henning, T., Hippler, S., Horrobin, M., Huber, A., Hubert, Z., Hubin, N., Hummel, C. A., Jakob, G., Janssen, A., Jochum, L., Jocou, L., Kaufer, A., Kellner, S., Kendrew, S., Kern, L., Kervella, P., Kiekebusch, M., Klein, R., Kok, Y., Kolb, J., Kulas, M., Lacour, S., Lapeyrère, V., Lazareff, B., Le Bouquin, J.-B., Lèna, P., Lenzen, R., Lévêque, S., Lippa, M., Magnard, Y., Mehrgan, L., Mellein, M., Mérand, A., Moreno-Ventas, J., Moulin, T., Müller, E., Müller, F., Neumann, U., Oberti, S., Ott, T., Pallanca, L., Panduro, J., Pasquini, L., Paumard, T., Percheron, I., Perraut, K., Perrin, G., Pflüger, A., Pfuhl, O., Phan Duc, T., Plewa, P. M., Popovic, D., Rabien, S., Ramírez, A., Ramos, J., Rau, C., Riquelme, M., Rohloff, R.-R., Rousset, G., Sanchez-Bermudez, J., Scheithauer, S., Schöller, M., Schuhler, N., Spyromilio, J., Straubmeier, C., Sturm, E., Suarez, M., Tristram, K. R. W., Ventura, N., Vincent, F., Waisberg, I., Wank, I., Weber, J., Wieprecht, E., Wiest, M., Wiezorrek, E., Wittkowski, M., Woillez, J., Wolff, B., Yazici, S., Ziegler, D., and Zins, G., "First light for GRAVITY: Phase referencing optical interferometry for the Very Large Telescope Interferometer," A\&A 602, A94 (June 2017).

[35] Peterson, B. M., "Variability of Active Galactic Nuclei," in [Advanced Lectures on the Starburst-AGN], Aretxaga, I., Kunth, D., and Mújica, R., eds., 3 (2001).

[36] Koshida, S., Minezaki, T., Yoshii, Y., Kobayashi, Y., Sakata, Y., Sugawara, S., Enya, K., Suganuma, M., Tomita, H., Aoki, T., and Peterson, B. A., "Reverberation Measurements of the Inner Radius of the Dust Torus in 17 Seyfert Galaxies," ApJ 788, 159 (June 2014).

[37] Peterson, B. M., Berlind, P., Bertram, R., Bischoff, K., Bochkarev, N. G., Borisov, N., Burenkov, A. N., Calkins, M., Carrasco, L., Chavushyan, V. H., Chornock, R., Dietrich, M., Doroshenko, V. T., Ezhkova, O. V., Filippenko, A. V., Gilbert, A. M., Huchra, J. P., Kollatschny, W., Leonard, D. C., Li, W., Lyuty, V. M., Malkov, Y. F., Matheson, T., Merkulova, N. I., Mikhailov, V. P., Modjaz, M., Onken, C. A., Pogge, R. W., Pronik, V. I., Qian, B., Romano, P., Sergeev, S. G., Sergeeva, E. A., Shapovalova, A. I., Spiridonova, O. I., Tao, J., Tokarz, S., Valdes, J. R., Vlasiuk, V. V., Wagner, R. M., and Wilkes, B. J., "Steps toward 
Determination of the Size and Structure of the Broad-Line Region in Active Galactic Nuclei. XVI. A 13 Year Study of Spectral Variability in NGC 5548," ApJ 581, 197-204 (Dec. 2002).

[38] Koshida, S., Yoshii, Y., Kobayashi, Y., Minezaki, T., Sakata, Y., Sugawara, S., Enya, K., Suganuma, M., Tomita, H., Aoki, T., and Peterson, B. A., "Variation of Inner Radius of Dust Torus in NGC4151," ApJ 700, L109-L113 (Aug. 2009).

[39] Pott, J.-U., Malkan, M. A., Elitzur, M., Ghez, A. M., Herbst, T. M., Schödel, R., and Woillez, J., "Luminosity-variation Independent Location of the Circum-nuclear, Hot Dust in NGC 4151," ApJ 715, 736-742 (June 2010).

[40] Kishimoto, M., Hönig, S. F., Antonucci, R., Millan-Gabet, R., Barvainis, R., Millour, F., Kotani, T., Tristram, K. R. W., and Weigelt, G., "Evidence for a Receding Dust Sublimation Region around a Supermassive Black Hole," ApJ 775, L36 (Oct. 2013).

[41] Elvis, M. and Karovska, M., "Quasar Parallax: A Method for Determining Direct Geometrical Distances to Quasars," ApJ 581, L67-L70 (Dec. 2002).

[42] Hönig, S. F., Watson, D., Kishimoto, M., and Hjorth, J., "A dust-parallax distance of 19 megaparsecs to the supermassive black hole in NGC 4151," Nature 515, 528-530 (Nov. 2014).

[43] Kraus, S., Monnier, J. D., Ireland, M. J., Duchêne, G., Espaillat, C., Hönig, S., Juhasz, A., Mordasini, C., Olofsson, J., Paladini, C., Stassun, K., Turner, N., Vasisht, G., Harries, T. J., Bate, M. R., Gonzalez, J.-F., Matter, A., Zhu, Z., Panic, O., Regaly, Z., Morbidelli, A., Meru, F., Wolf, S., Ilee, J., Berger, J.-P., Zhao, M., Kral, Q., Morlok, A., Bonsor, A., Ciardi, D., Kane, S. R., Kratter, K., Laughlin, G., Pepper, J., Raymond, S., Labadie, L., Nelson, R. P., Weigelt, G., ten Brummelaar, T., Pierens, A., Oudmaijer, R., Kley, W., Pope, B., Jensen, E. L. N., Bayo, A., Smith, M., Boyajian, T., Quiroga-Nuñez, L. H., Millan-Gabet, R., Chiavassa, A., Gallenne, A., Reynolds, M., de Wit, W.-J., Wittkowski, M., Millour, F., Gandhi, P., Ramos Almeida, C., Alonso Herrero, A., Packham, C., Kishimoto, M., Tristram, K. R. W., Pott, J.-U., Surdej, J., Buscher, D., Haniff, C., Lacour, S., Petrov, R., Ridgway, S., Tuthill, P., van Belle, G., Armitage, P., Baruteau, C., Benisty, M., Bitsch, B., Paardekooper, S.-J., Pinte, C., Masset, F., and Rosotti, G., "Planet Formation Imager (PFI): science vision and key requirements," in [Optical and Infrared Interferometry and Imaging V], Proc. SPIE 9907, 99071K (Aug. 2016). 